\title{
ERRATUM
}

K. E. Arendt · S. H. Jónasdóttir · P. J. Hansen

S. Gärtner

\section{Effects of dietary fatty acids on the reproductive success of the calanoid copepod Temora longicornis}

Published online: 16 February 2006

(C) Springer-Verlag 2006

\section{Marine Biology (2005) 146:513-530}

In Table 3, column "Phaeocystis globosa" the value for "Cells (ml-1 initial concentration" was wrong and consequently led to other miscalculated values. The corrected Table 3 is shown below. The error did not affect subsequent results or conclusions of the paper.

Table 3 Temora longicornis fed T. weissflogii, P. globosa, Isochrysis sp. and $D$. tertiolecta. Only data from experiments in which the reduction of the initial prey concentration was between 5 and $30 \%$ are given. Carbon content of T. longicornis females of $12.6 \mu \mathrm{g}$ was calculated from data by Klein Breteler and Gonzalez (1988) and
Kiørboe and Nielsen (1994). Carbon-based gross growth efficiency $\left(\mathrm{GGE}_{\mathrm{C}}\right)$ was calculated by using a carbon content of $T$. longicornis eggs $\left(C_{\text {egg }}=0.0883 \mu \mathrm{g}\right)$ from Dam and Lopes (2003). Values are means \pm 1 standard deviation ( $n$ number of replicates)

\begin{tabular}{|c|c|c|c|c|}
\hline & Thalassiosira. weissflogii & Phaeocystis globosa & Isochrysis sp. & Dunaliella tertiolecta \\
\hline Clearance rate & $n=8$ & $n=12$ & $n=5$ & $n=7$ \\
\hline Volume $\left(\mathrm{ml}\right.$ animal $\left.{ }^{-1} \mathrm{~h}^{-1}\right)$ & $1.56 \pm 0.58$ & $0.29 \pm 0.23$ & $0.49 \pm 0.14$ & $0.21 \pm 0.19$ \\
\hline Cells $\left(\right.$ animal $\left.^{-1} \mathrm{~h}^{-1}\right)$ & $8,481 \pm 2,919$ & $92,580 \pm 63,977$ & $154,217 \pm 57,348$ & $2,426 \pm 2,277$ \\
\hline Carbon $\left(\mu \mathrm{g}\right.$ animal $\left.^{-1} \mathrm{~h}^{-1}\right)$ & $0.65 \pm 0.2$ & $0.86 \pm 0.6$ & $0.80 \pm 0.4$ & $0.10 \pm 0.1$ \\
\hline Lipid $\left(\mu \mathrm{g} \operatorname{animal}^{-1} \mathrm{~h}^{-1}\right)$ & 0.082 & 0.09 & 0.188 & 0.016 \\
\hline $\mathrm{C}_{\text {egg }}\left(\mu \mathrm{g} \mathrm{female} \mathrm{f}^{-1} \mathrm{~h}^{-1}\right)$ & 0.10 & 0.08 & 0.13 & 0.01 \\
\hline $\mathrm{GGE}_{\mathrm{C}}$ & 0.16 & 0.23 & 0.16 & 0.09 \\
\hline Cells (ml-1, initial concentration) & 4,587 & 305,625 & 248,510 & 19,100 \\
\hline$\mu \mathrm{g} \mathrm{C}^{-1}$ (initial concentration) & 349 & 2,842 & 2,038 & 916 \\
\hline Days used for $E_{\mathrm{r}}$ average & $3-6$ & $4-6$ & $4-6$ & $4-6$ \\
\hline
\end{tabular}

The online version of the original article can be found at http:// dx.doi.org/10.1007/s00227-004-1457-9

K. E. Arendt · S. H. Jónasdóttir $(\bowtie) \cdot S$. Gärtner Department of Marine Ecology and Aquaculture, Danish Institute for Fisheries Research,

Kavalergaarden 6, 2920, Charlottenlund, Denmark

E-mail: sjo@dfu.min.dk

Tel.: + 45-3396-3427

Fax: + 45-3396-3434

P. J. Hansen

Marine Biological Laboratary, University of Copenhagen,

Strandpromenaden 5, 3000, Helsingør, Denmark 